\title{
Cervical Oesophagotomy and Removal of an Impacted Oesophageal Denture: Case Report and Literature Review
}

\author{
Isaac Okyere1,2*, Sanjeev Singh ${ }^{2,3}$, Perditer Okyere ${ }^{2,4}$, Antwi Kusi2,3, Samuel Gyasi Brenu1 \\ ${ }^{1}$ Cardiovascular and Thoracic Surgery Unit, Directorate of Surgery, Komfo Anokye Teaching Hospital, Kumasi, Ghana \\ ${ }^{2}$ School of Medicine and Dentistry, College of Health Sciences, Kwame Nkrumah University of Science and Technology, \\ Kumasi, Ghana \\ ${ }^{3}$ Directorate of Anaesthesia and Intensive Care, Komfo Anokye Teaching Hospital, Kumasi, Ghana \\ ${ }^{4}$ Renal Unit, Directorate of Medicine, Komfo Anokye Teaching Hospital, Kumasi, Ghana \\ Email: *drokyere@yahoo.com
}

How to cite this paper: Okyere, I., Singh, S., Okyere, P., Kusi, A. and Brenu, S.G. (2020) Cervical Oesophagotomy and Removal of an Impacted Oesophageal Denture: Case Report and Literature Review. Surgical Science, 11, 177-186.

https://doi.org/10.4236/ss.2020.117020

Received: May 11, 2019

Accepted: June 30, 2020

Published: July 3, 2020

Copyright $(2020$ by author(s) and Scientific Research Publishing Inc. This work is licensed under the Creative Commons Attribution International License (CC BY 4.0).

http://creativecommons.org/licenses/by/4.0/

\begin{abstract}
We present the case of a 46-year old man with a two-year history of losing his denture presenting with cachexia and a two-month history of dysphagia to both liquids and solids associated with odynophagia. Review at the ENT department where a diagnostic rigid oesophagoscopy was done under general anaesthesia showed a denture impacted to the wall of the oesophagus at 17 $\mathrm{cm}$ from the incisor. Attempts at retrieval were unsuccessful as the denture was deeply embedded in the oesophageal wall and bled easily on attempted removal. He subsequently underwent a 2 -stage surgical management approach by the cardiothoracic surgical team. Patient first underwent nutritional rehabilitation for a month through a created feeding gastrostomy tube via a limited midline laparotomy. He then had a open cervical oesophagotomy and removal of the denture. Currently doing well six months after surgery and tolerating normal oral diet.
\end{abstract}

\section{Keywords}

Impacted Oesophageal Denture, Surgery, Cervical Oesophagotomy, Feeding Gastrostomy

\section{Introduction}

Dentures have been worn by patients for generations. The plastic material from which present-day dentures are made is radiolucent making their diagnosis notoriously challenging by imaging. Although the use of radiopaque substances for 
denture manufacturing has been recommended, such material is yet to be successfully used without compromising denture quality. Hence, there is often a delay in the diagnosis and management of patients with impacted dentures, predisposing them to higher risks of complications such as oesophageal perforation, diverticulum, fistula formation and bowel obstruction. Several complications from impacted dentures have been published but the literature is sparse on impacted dentures causing an oesophageal diverticulum. We present a case of an impacted denture causing a distal cervical oesophageal diverticulum who underwent a successful surgical removal through cervical oesophagotomy after failed attempted removal by rigid oesophagoscopy.

\section{Case Report}

A 46-year old male who has had a denture fixed 13 years earlier was well until one morning when he noticed he was missing his denture upon waking up and this was two years prior to presentation to our centre. He reported to several peripheral hospitals where X-rays done as usual did not visualize the denture and as such was reassured and discharged home. He was well until two months prior to presentation when he started experiencing dysphagia initially to solids and subsequently to both solids and liquids. Symptoms were associated with odynophagia and hoarseness of voice with significant unmeasured weight loss. He was referred to the ENT department where a diagnostic rigid oesophagoscopy was done under general anaesthesia. A denture was found impacted to the wall of the oesophagus at $17 \mathrm{~cm}$ from the incisor and attempts at retrieval were unsuccessful as the denture was deeply embedded in the oesophagus. The patient was subsequently referred to cardiothoracic surgery.

On review, he was cachectic and chronically dehydrated but hemodynamically stable. Laboratory investigations were significant for Serum Albumin of $24 \mathrm{~g} / \mathrm{L}$ $(35-50 \mathrm{~g} / \mathrm{L})$. He was optimized and prepared for a Stamm feeding gastrostomy for nutritional rehabilitation as he was not nutritionally fit for major surgery. $\mathrm{He}$ was then discharged home to continue nutritional rehabilitation whilst being worked up for surgical removal.

He was readmitted a month later having significantly gained weight. Chest $\mathrm{X}$-ray as shown in Figure 1, did not visualize any foreign body in the oesophagus, areas of consolidation in the lung fields or pleural collection. Contrast study of the oesophagus by Barium Swallow as shown in Figure 2, showed a proximal oesophageal stricture at T1 - T2 with multiple proximal oesophageal pseudodiverticula but no tracheoesophageal fistula. All haematological and biochemical investigations were within normal limits.

With obtained informed consent, patient was sent to the operating theatre for an exploratory cervical oesophagotomy and denture removal. Under general anaesthesia and cuffed endotracheal intubation, a c-collar incision at the base of the neck was made and by careful blunt and sharp dissection on the left lateral aspect of the trachea, the oesophagus was isolated at the level of C7 - T1. A $4 \mathrm{~cm}$ 


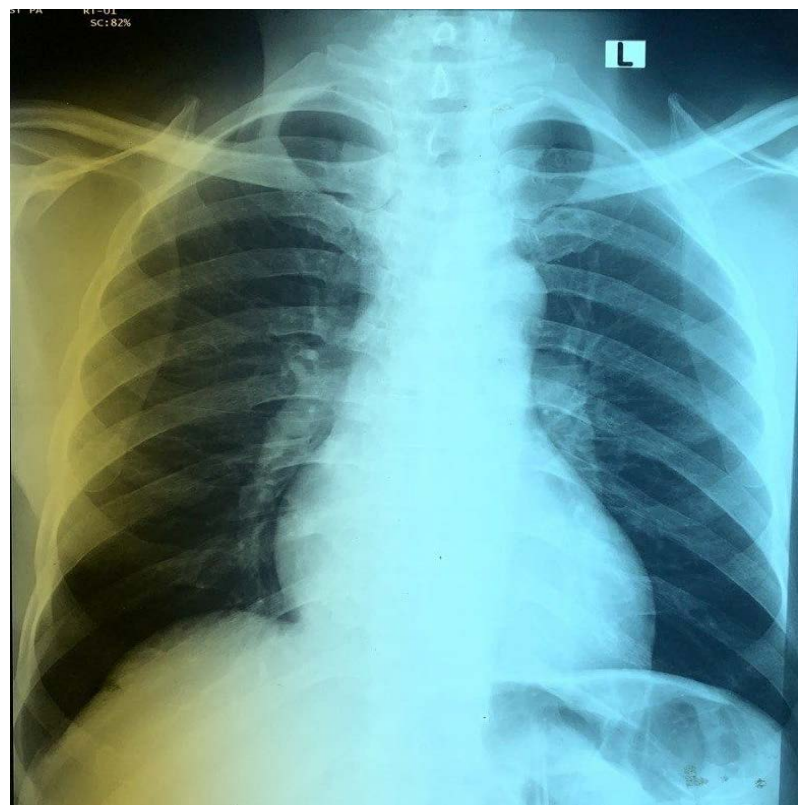

Figure 1. Chest-ray showing relatively normal findings.

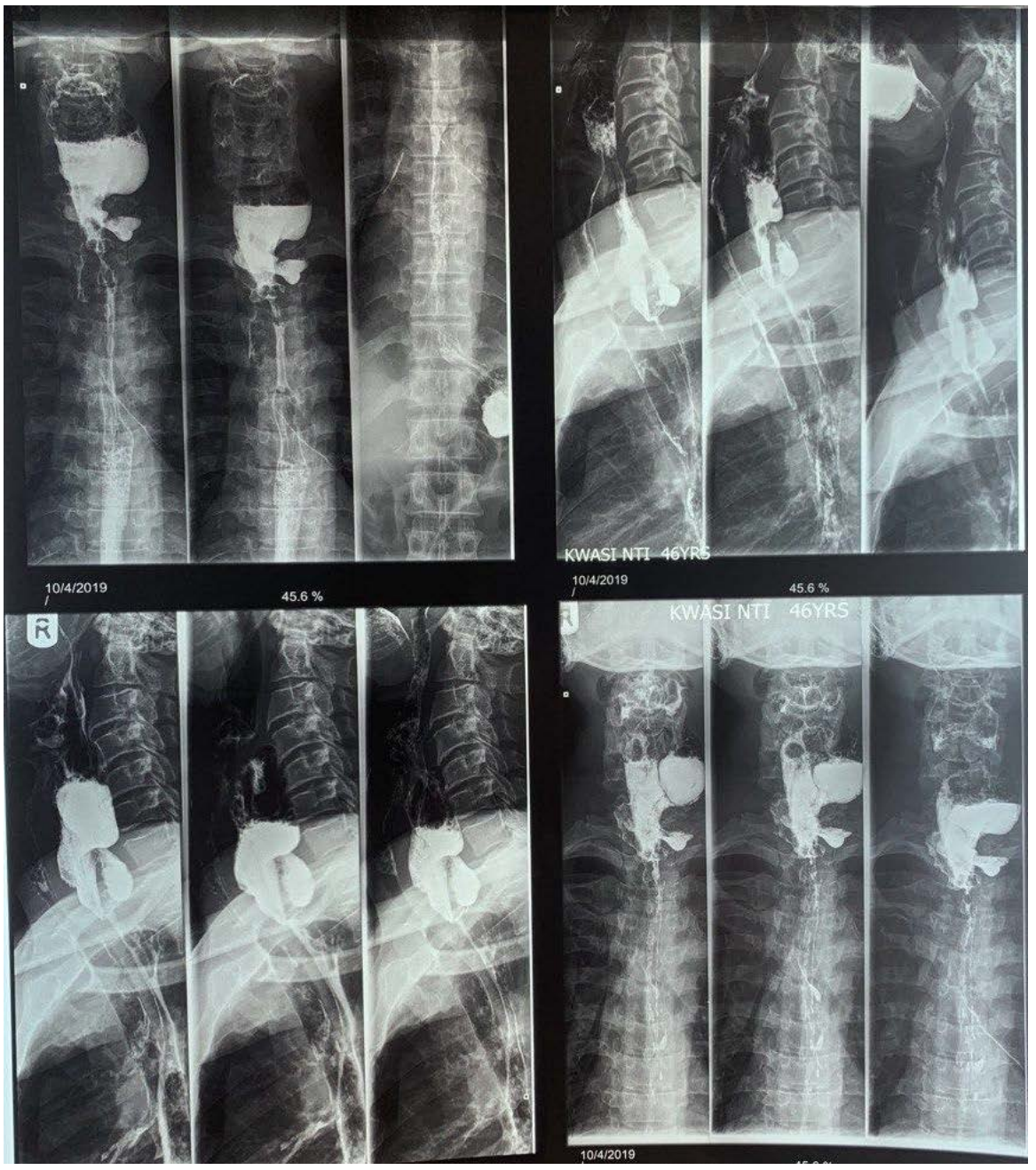

Figure 2. Preoperative contrast study showing the multiple diverticuli. 
longitudinal incision was made over the palpated denture and the impacted denture was carefully removed en bloc as depicted by the intraoperative photo of Figure 3. After inspection of the posterior oesophageal wall for any perforation and as shown in Figure 4 and Figure 5, the oesophagotomy was repaired in an interrupted double-layered suturing with vicryl $3 / 0$ after normal saline irrigation and the neck incision was then closed up in layers.

Patient was kept nil by mouth for 7 days but resumed feeding through the gastrostomy tube on postoperative day 2. A repeat water-soluble contrast study done on postoperative day 8 as shown in Figure 6, showed a smooth narrowing

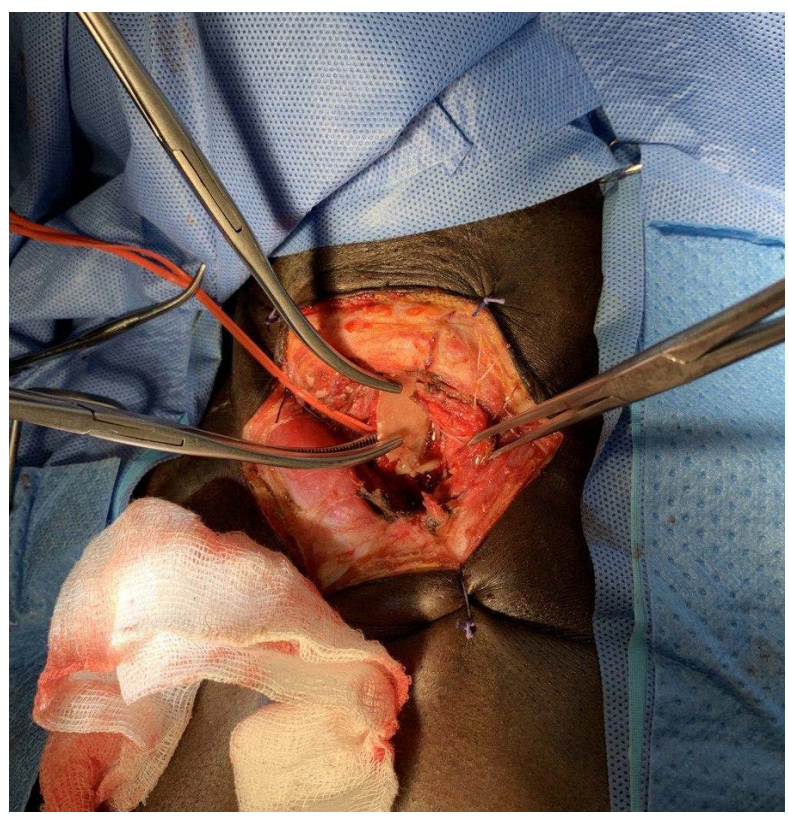

Figure 3. Impacted denture being carefully retrieved.

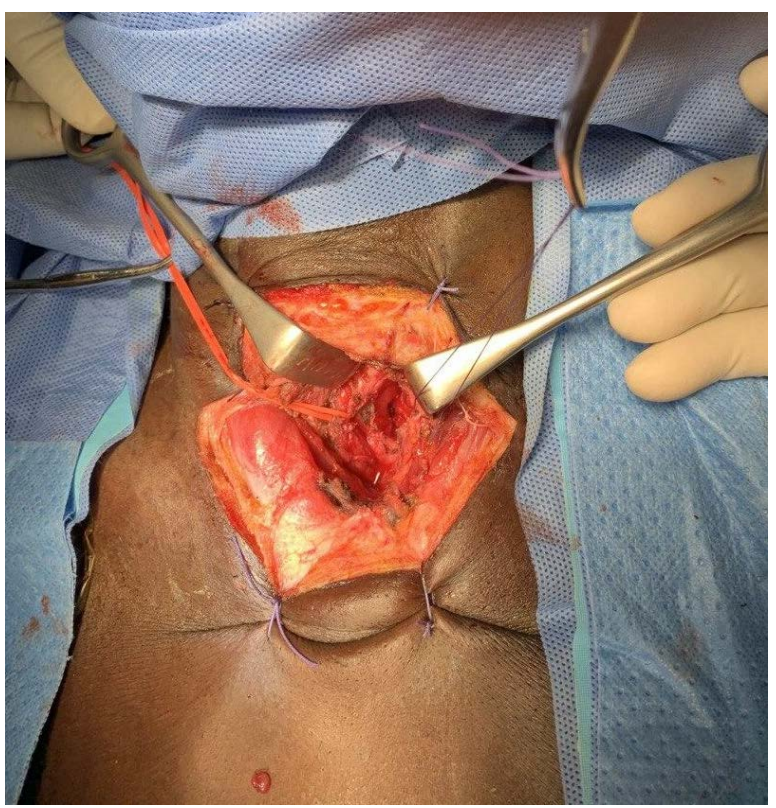

Figure 4. Showing the oesophagus after denture removal. 


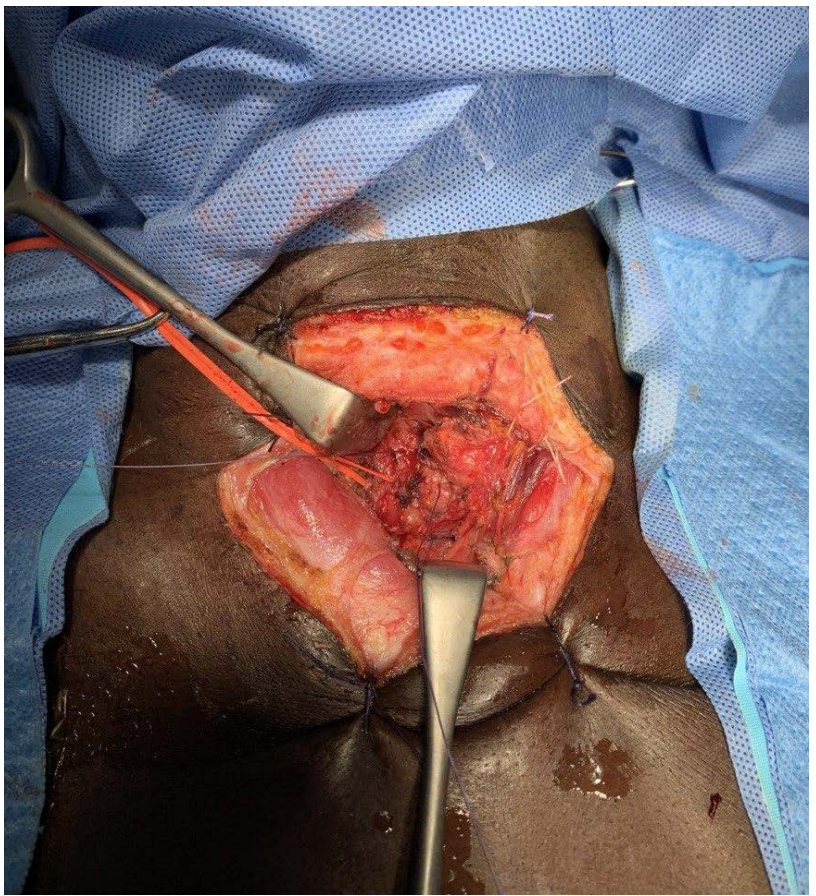

Figure 5. Picture of repaired oesophagotomy.

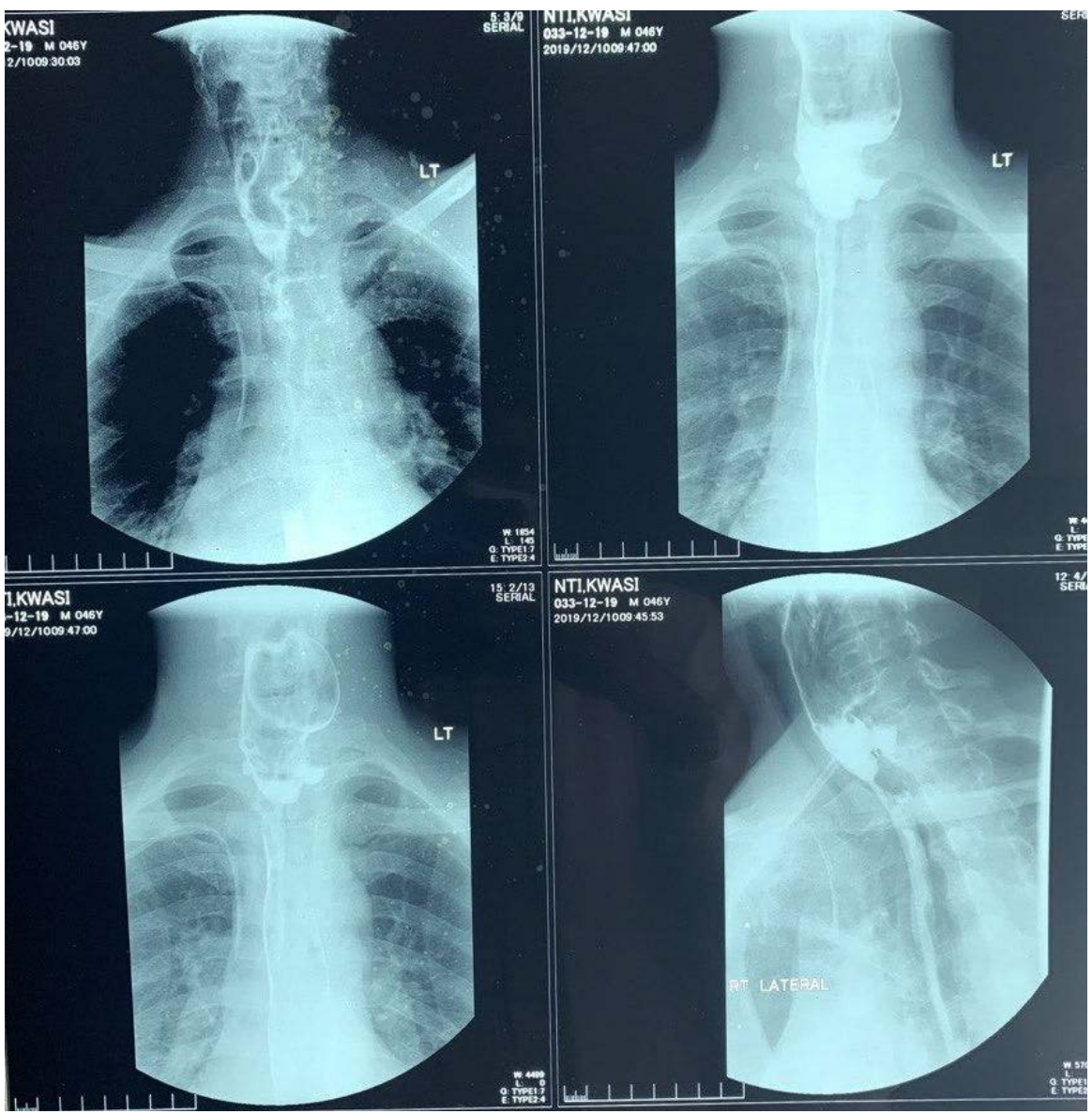

Figure 6. Postoperative contrast oesophagogram showing the patent but proximally dilated oesophagus. 
of the proximal oesophagus at T1 level with evidence of pre-stenotic dilatation but contrast seen to flow beyond that level into the distal oesophagus. No evidence of fistulous tract, outpouching or extravasation of contrast seen. The patient was then started on graduated oral intake on postoperative day 10 which he tolerated. He was subsequently discharged home on postoperative day 14 after removal of the feeding gastrostomy tube and been followed up on out-patient basis, reviewed after two weeks, one month, then in three months. Figure 7 shows his healed collar incision wound and doing well six months after surgery tolerating normal diet.

\section{Discussion}

Missing dentition in humans is a particularly upsetting condition since it is aesthetically displeasing, significantly diminishes one's self-confidence and also impacts food choices. As such the need for replacement of lost dentition is paramount. This is to restore aesthetic defect and nutritional function. Dentures are acrylic-based models of the lost dentition designed to fill the gaps left by the missing teeth [1]. Concomitantly, there has been increase in the prevalence of accidental denture ingestion with the most common place of impaction in most cases being the oesophagus [2]. Oesophageal denture impaction is most prevalent among the elderly due to their impaired oral mucosal sensitivity and pharyngeal motor control [2] [3] [4]. It has been quoted to contribute about $18 \%$ of cases of oesophageal foreign bodies as reported by Samargandy et al. [5]. Male gender has been found to have a high prevalence than females despite the fact that more females tend to have dentures than males. Adedeji and friends found that majority of their patients with impacted oesophageal dentures were male [1]. Our patient was a male of relatively young age of 46 years though a previous paper by Isaac Okyere et al. had reported from Ghana of a 6-year old child with an impacted coin in the mid-oesophagus which had been retrieved by open
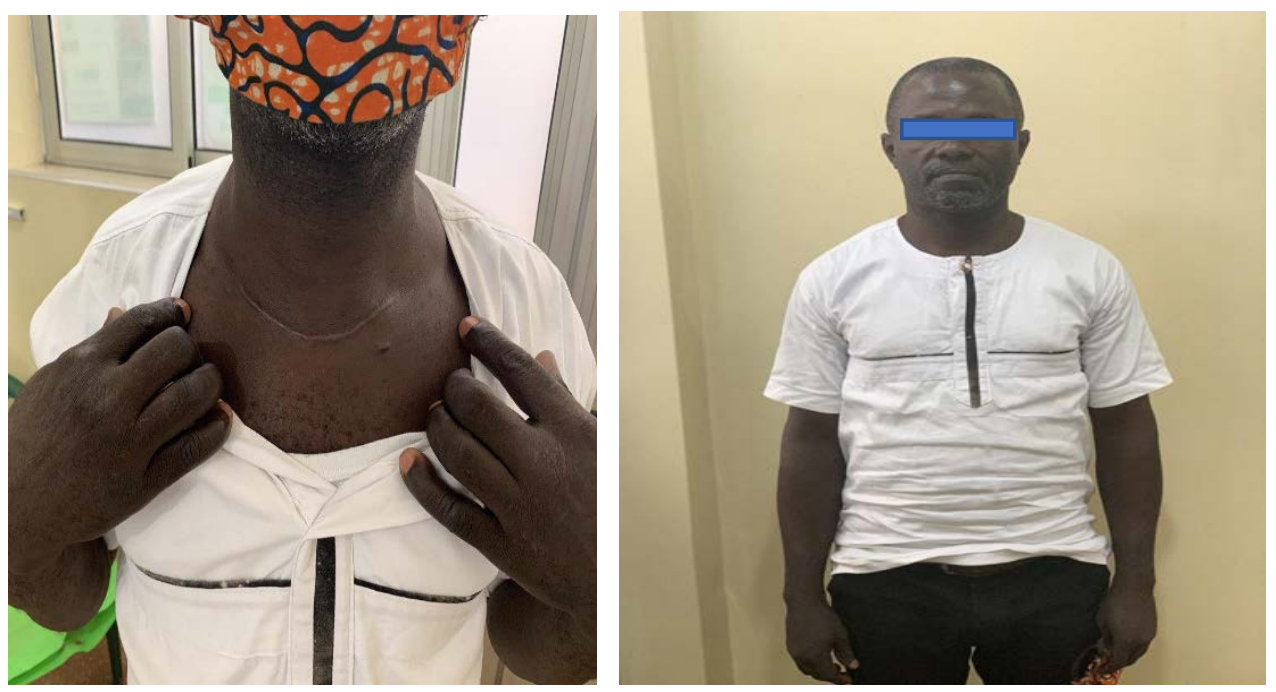

Figure 7. Healed c-collar incision and patient after 6 months. 
thoracic surgery via right thoracotomy after failed attempted removal via rigid esophagoscopy [6].

Conditions predisposing to denture ingestion include maxillofacial trauma, cerebrovascular accident, alcohol intoxication and psychiatric illness. Conscious patients are also prone to denture ingestion during falls, sleep and anaesthesia [2]. Our patient woke up from sleep and found the denture missing. A systematic review of literature done by Khalfallah and colleagues showed that in about half of the 18 case reports on impacted oesophageal dentures reviewed, ingestion occurred during swallowing while less than $10 \%$ of the cases occurred after an impact to the face. Two out of the 18 cases occurred during sleep [7] which is similar to our case. However, some cases may not be associated with any of the stated risk factors but may rather be a dental complication where the dentures may be loose or damaged [5].

Majority of denture impactions occur in areas of physiological constrictions or stenoses, with the oesophagus being the most common site. The cricopharyngeal constriction at $15 \mathrm{~cm}$ from the incisors being the narrowest part of the oesophagus serves as the commonest site of denture impaction [2] as was the case in our patient. However, it may also lodge in the mid-oesophagus at the tracheal bifurcation or the distal oesophagus at the lower oesophageal sphincter [8] as was reported in the case of Isaac Okyere et al. from Ghana [6]. A retrospective study by Okugbo and Onyeagwara in Nigeria discovered that all but 2 out of 44 cases reviewed had the dentures impacted above the tracheal bifurcation [8].

\section{Clinical Presentation and Complications}

Foreign body ingestions typically traverse the gastrointestinal tract and are expelled spontaneously. However, in the event of oesophageal impaction, the most common symptom is dysphagia as depicted by multiple literatures [4] [6] [7]. Other concomitant symptoms include odynophagia, neck pain, chest pain and regurgitation [4] [5] [6] [7]. Some patients may be initially asymptomatic following ingestion and as such predispose to delayed presentation which can precipitate the development of complications [4]. This is the situation of our patient who missed his denture two years before presenting with complications of diverticula with dysphagia to solids and liquids, weight loss and odynophagia being the main symptomatology.

Possible complications that can arise from denture impaction include oesophageal perforation and mediastinitis, retropharyngeal abscesses, tracheoesophageal fistula, aorto-oesophageal fistula and pneumomediastinum [6]. Samargandy et al. showed that the rate of complications rose from $3.2 \%$ to $23.5 \%$ when patients who were managed within 24 hours of ingestion of denture were compared with those managed after 48 hours of ingestion. The case report published by them reported of an impacted denture which had been in situ for about 2 years with subsequent diverticulum formation [5], similar to our case report. Tamatey and colleagues in 2008 also reported of a rare case of a 48 -year old male who developed cervical oesophageal diverticulum from an impacted one tooth partial 
denture [9].

\section{Diagnosis}

Dentures are commonly manufactured from methlymethacrylate or porcelain, materials which are radiolucent. As such the use of X-rays in diagnosis is usually non-informative when the impacted denture is made of any of these materials. In a study done in Nigeria, none out of the 44 patients reviewed had their dentures detected on X-ray [8]. However, possible signs of X-ray which may be suggestive of possible denture impaction in the oesophagus include thickened prevertebral soft tissues, air in the oesophagus, presence of foreign body shadow in oesophagus and air-fluid level in oesophagus. In a bid to improve visibility on X-rays, some dentures have metal hinges fixed to them [2] [4] [5] [8]. X-rays, however, are useful in detecting impaction-associated complications such as pneumomediastinum, pneumothorax, subcutaneous emphysema or hydrothorax [2]. CT scans offer better visibility of acrylic dentures than X-rays and also provides better diagnostic accuracy for potential complications [2] [4].

Water-soluble oesophagogram is helpful in detecting the location of impacted denture and also map out any diverticulum or fistula complicating it [5]. However, it is not advisable to be carried out just prior to endoscopic retrieval since it coats the denture and renders removal challenging [1] [5].

The primary investigation for diagnosis is oesophagoscopy, either flexible or rigid. It is invaluable as it affords the opportunity to directly visualize the denture and possibly allow its immediate removal [8]. Our patient underwent examination under anaesthesia by rigid oesophagoscopy which detected a denture at $17 \mathrm{~cm}$ from the incisor. However, there was failure of attempted removal. Patient had barium swallow after this as part of pre-operative assessment to detect any associated complications such as diverticula, perforations and fistulae.

\section{Management}

Prompt removal of the denture as soon as diagnosis is made is of paramount importance due to the progressively increasing risk of complications with each day of delay. Options for retrieval are endoscopic and surgical removal [4] [6] [8] [10]. Endoscopic removal, either by flexible or rigid oesophagoscopy under general anaesthesia, is the preferred option [2]. The retrospective review done by Adedeji and colleagues showed over $70 \%$ success rate in the use of rigid esophagoscopy in retrieving impacted dentures [1]. Mizuno et al. (2016) also reported of 27 oesophagoscopies with $92 \%$ success rates in their retrospective study done in Japan. Similarly high success rates have been reported in Nigeria by Okugbo and Onyeagwara in 2012 [8]. Endoscopic retrieval of dentures is not devoid of complications due to the sharp edges and metal hinges attached to them. The procedure is associated with a high risk of oesophageal perforation and as such considerable level of skill and patience is required [2] [10].

Failure of endoscopic removal or in the advent of a contraindication to endoscopic removal, an open surgical approach to denture retrieval should be explored. The approach that is either cervical or trans-thoracic oesophagotomy 
may be opted for, depending on the location of the impacted denture [1] [2] [6] [8] [10]. A case report by Chua and friends described a surgical removal of a denture via cervical oesophagotomy and primary closure [10] similar to our case. Oesophagotomy is also associated with a potentially fatal complication of mediastinitis from oesophageal leakage from breakdown of the repaired oesophagotomy. This is associated with high mortality [10]. Oesophago-cutaneous fistula was documented to have been a complication of the case report by Dörner and colleagues in 2006 though fistula healed successfully after 3 weeks of conservative treatment [11]. Some cases of oesophago-cutaneous fistula may not be amenable to conservative management and as such may require re-exploration as was seen in the publication by Tamatey et al. [9]. In extreme cases of non-viable oesophageal tissues, oesophageal replacement with a colon bypass might be required to restore luminal continuity [8] [9]. Our patient presented with multiple diverticulae as a complication of the impacted denture and he was nutritionally incompetent due to moderate weight loss, therefore he was nutritionally rehabilitated through the creation of feeding tube gastrostomy through limited upper midline laparotomy for over a month before he was readmitted and stabilised for open surgery. He had cervical oesophagotomy through a collar incision at the base of the neck for the denture removal. Previously, Isaac Okyere et al. had reported of their successful management of open surgery retrieval of an impacted coin which has stayed for over two years at the mid-oesophagus and had to be removed through right thoracotomy and oesophagotomy [6].

\section{Conclusion}

When managing certain patients with dysphagia and odynophagia, a high index of suspicion for foreign body ingestion is needed even with prolonged symptoms. Emphasis on enquiring about dentures is particularly of value especially in the elderly, and thorough examination of the oral cavity and dentition is essential. Even though most dentures are radiolucent, diagnostic studies including upper gastrointestinal endoscopy, Barium Oesophagogram and CT scan of the neck and chest are still essential. Lateral neck radiographs might be useful in detecting radiopaque clasps on dental prostheses. Though endoscopic removal is the standard modality of treatment, successful open surgery removal is possible in the event of failed or contraindication to endoscopic removal.

\section{Acknowledgements}

Written informed consent was obtained from the patient for publication of the case and accompanying images and we are grateful to the theatre staff of the cardiovascular and thoracic surgery unit of the department of surgery at the Komfo Anokye Teaching Hospital.

\section{Conflicts of Interest}

The authors declare no conflicts of interest regarding the publication of this paper. 


\section{References}

[1] Adedeji, T.O., Olaosun, A.O., Sogebi, O.A. and Tobih, J.E. (2014) Denture Impaction in the Oesophagus Experience of a Young ENT Practice in Nigeria. The Pan African Medical Journal, 18, Article 330. https://doi.org/10.11604/pamj.2014.18.330.2871

[2] Gachabayov, M., Isaev, M., Orujova, L., Isaev, E., Yaskin, E. and Neronov, D. (2015) Swallowed Dentures: Two Cases and a Review [Internet]. Annals of Medicine and Surgery, 4, 407-413. https://doi.org/10.1016/j.amsu.2015.10.008

[3] Mizuno, K.I., Takahashi, K., Tominaga, K., Nishigaki, Y., Sato, H., Ikarashi, S., et al. (2016) Endoscopic Removal of Ingested Dentures and Dental Instruments: A Retrospective Analysis. Gastroenterology Research and Practice, 2016, Article ID: 3537147. https://doi.org/10.1155/2016/3537147

[4] Singh, P., Singh, A., Kant, P., Zonunsanga, B. and Singh Kuka, A. (2013) An Impacted Denture in the Oesophagus-An Endoscopic or a Surgical Emergency-A Case Report. Journal of Clinical and Diagnostic Research, 7, 919-920. https://doi.org/10.7860/JCDR/2013/5337.2976

[5] Samargandy, S., Marzouki, H., Al-Khatib, T. and Merdad, M. (2019) Denture Impaction Causing an Upper Esophageal Diverticulum. Case Reports in Gastrointestinal Medicine, 2019, Article ID: 9621383. https://doi.org/10.1155/2019/9621383

[6] Okyere, I., Brenu, S.G. and Okyere, P. (2020) Open Thoracic Surgery for Removal of a Two-Year-Old Impacted Esophageal Coin. Open Journal of Thoracic Surgery, 10, 32-40. https://doi.org/10.4236/ojts.2020.101004

[7] Khalfallah, M., et al. (2011) Denture Foreign Bodies Impacted in the Thoracic Esophagus. A Systematic Review. Revista Portuguesa de Cirurgia, 16, 13-19.

[8] Okugbo, S.U. and Onyeagwara, N.C. (2012) Oesophageal Impacted Dentures at the University of Benin Teaching Hospital, Benin City, Nigeria. Journal of the West African College of Surgeons, 2, 85-94.

[9] Tamatey, M., Sereboe, L., Tettey, M., Edwin, F., Entsua-Mensah, K., Aniteye, E., Kotei, D., Delia, I., Okyere, I. and Frimpong-Boateng, K. (2008) Cervical Oesophageal Diverticulum Due to a Swallowed Impacted Denture: A Case Report. African Annals of Thoracic and Cardio-Vascular Surgery, 3, 12-14.

[10] Chua, Y.K.D., See, J.Y. and Ti, T.K. (2006) Oesophageal-Impacted Denture Requiring Open Surgery. Singapore Medical Journal, 47, 820-821.

[11] Dörner, J., Spelter, H., Zirngibl, H. and Ambe, P.C. (2017) Surgical Retrieval of a Swallowed Denture in a Schizophrenic Patient: A Case Report. Patient Safety in Surgery, 11, Article No.: 28. https://doi.org/10.1186/s13037-017-0147-8 